\title{
DISCUSSÕES SOBRE O DESENVOLVIMENTO E UTILIZAÇÃO DE UM PROTÓTIPO DIDÁTICO PARA ENSINO DE REDES INDÚSTRIAIS
}

\author{
DOI: 10.37702/2175-957X.COBENGE.2021.3485
}

Rômulo Lira Milhomem - romulo.milhomem@ifsc.edu.br

IFSC

89813-000 3450D

89813-000 - Chapecó - SC

Kimberly Vanessa da Hora Brandão Blum - kimberly.eng2016@gmail.com Instituto Federal Catarinense

Rua Mavalda Lingner Porto 93

89163-644 - Rio do Sul - SC

Resumo: A necessidade de profissionais habilitados a trabalharem com as tecnologias para automação de processos industriais vem crescendo e se tornando cada vez mais imperativa devido a integração das informações e a crescente informatização desses processos. Neste caso, faz-se necessário uma formação técnica de qualidade que desenvolva a integração pratica e teórica dos conhecimentos de forma atrativa. Neste contexto, foi construído um protótipo de Bancada de Redes Industriais, objetivando melhor a apresentação de conhecimentos no ensino de automação, integrando as disciplinas de Redes de Comunicação e programação de Controladores Lógicos Programáveis. Por fim discute-se sobre a construção de protótipos como a Bancada de Redes Industriais, como forma didática e atraente de ensino técnico, apresentando os futuros profissionais a suas possíveis rotinas de trabalho.

Palavras-chave: Bancada de Redes Industriais; Intergeração de Sistemas; Protótipo Didático. 


\section{DISCUSSÕES SOBRE O DESENVOLVIMENTO E UTILIZAÇÃO DE UM PROTÓTIPO DIDÁTICO PARA ENSINO DE REDES INDÚSTRIAIS}

\section{INTRODUÇÃO}

O ensino profissionalizante vem passando por constantes transformações, buscando tornar-se mais atrativo ao estudante, procurando atribuir sentido aos conteúdos e as transformações das informações procedentes dos diferentes conceitos. Assim, de acordo com as novas Diretrizes Curriculares Nacionais (DCNs) aplicadas a cursos de graduação em engenharia, deve-se estimular o uso de metodologias ativas para aprendizagem, como forma de promover uma educação mais centrada no estudante (BRASIL, 2019). Deste modo, o emprego de métodos de ensino que se fundamentam no uso contextualizado de uma situação problema, são de grande utilidade para 0 aprendizado autodirigido (BENDER, 2014).

Nesse contexto, a construção de protótipos como ferramentas didáticas é considerada uma boa metodologia de aprendizagem em cursos da área de tecnologia, já que para o desenvolvimento destas atividades necessita-se o a construção do raciocínio logico, embasamentos teóricos e habilidades técnicas do estudante, adquiridas em diversas disciplinas ao longo do curso, e integradas rumo a um resultado definido e solido. Neste caso, esta temática apresenta-se como uma boa solução para fundamentação de conhecimentos teóricos abordados em sala.

Assim, o objetivo do uso e construção de protótipos como ferramentas didáticas, neste caso, é simular processos encontrados em ambientes de trabalho que, normalmente, os estudantes egressos da área de automação serão inseridos, como: programação de controladores, configuração de redes de comunicação, montagem de circuitos eletrônicos e integração de sistemas de automação. De tal modo, objetiva-se apresentar o desenvolvimento de um protótipo, que pode ser empregado como uma ferramenta didática para ensino de engenharia de automação, a partir de experimentos voltados à configuração e programação de redes de comunicação industrial. Este protótipo foi denominado Bancada de Redes Industriais, o qual consiste em uma esteira ajustada para separar dois tipos de peças, de acordo com o tipo de material.

Para isso, o algoritmo de funcionamento do processo é programado em um Controlador Lógico Programável (CLP), assim como a configuração da rede de comunicação, a partir da configuração de gateways e módulos de entrada e saída remotos. Nesse sentido, na Seção 2, serão apresentados inicialmente os aspectos construtivos do protótipo desenvolvido. Na Seção 3, apresentam-se a configuração das redes de comunicação em conjunto com a lógica de programação do CLP, como exemplo de simulação de uma tarefa exercida pelo engenheiro de automação. Por fim, na Seção 4 os resultados e contribuições para o ensino em engenharia de automação são discutidos.

\section{ASPECTOS CONSTRUTIVOS}

A construção e o uso de protótipos didáticos levam os estudantes envolvidos a desenvolverem competências técnicas e interpessoais voltadas à aplicação prática dos conhecimentos agregados em sala de aula e o desenvolvimento de trabalho/atividades em equipe, introduzindo-os a uma realidade mais próxima do mundo do trabalho, empregando uma metodologia ativa de Aprendizagem Baseada em Projetos (PBL - do 
inglês Project Based Learning) para sintetizar os conhecimentos envolvidos no projeto pelo discente (BENDER, 2014).

Deste modo, foi construída a Bancada de Redes Industriais, que consiste em sensores e atuadores interligados com componentes de redes industriais, a fim de realizar a conversão de sinais provenientes do processo em informações para a programação realizada pelo CLP. Como demonstra o diagrama da Figura 1, que apresenta a interligação e o funcionamento geral do processo.

Figura 1 - Diagrama interligação e funcionamento do protótipo.

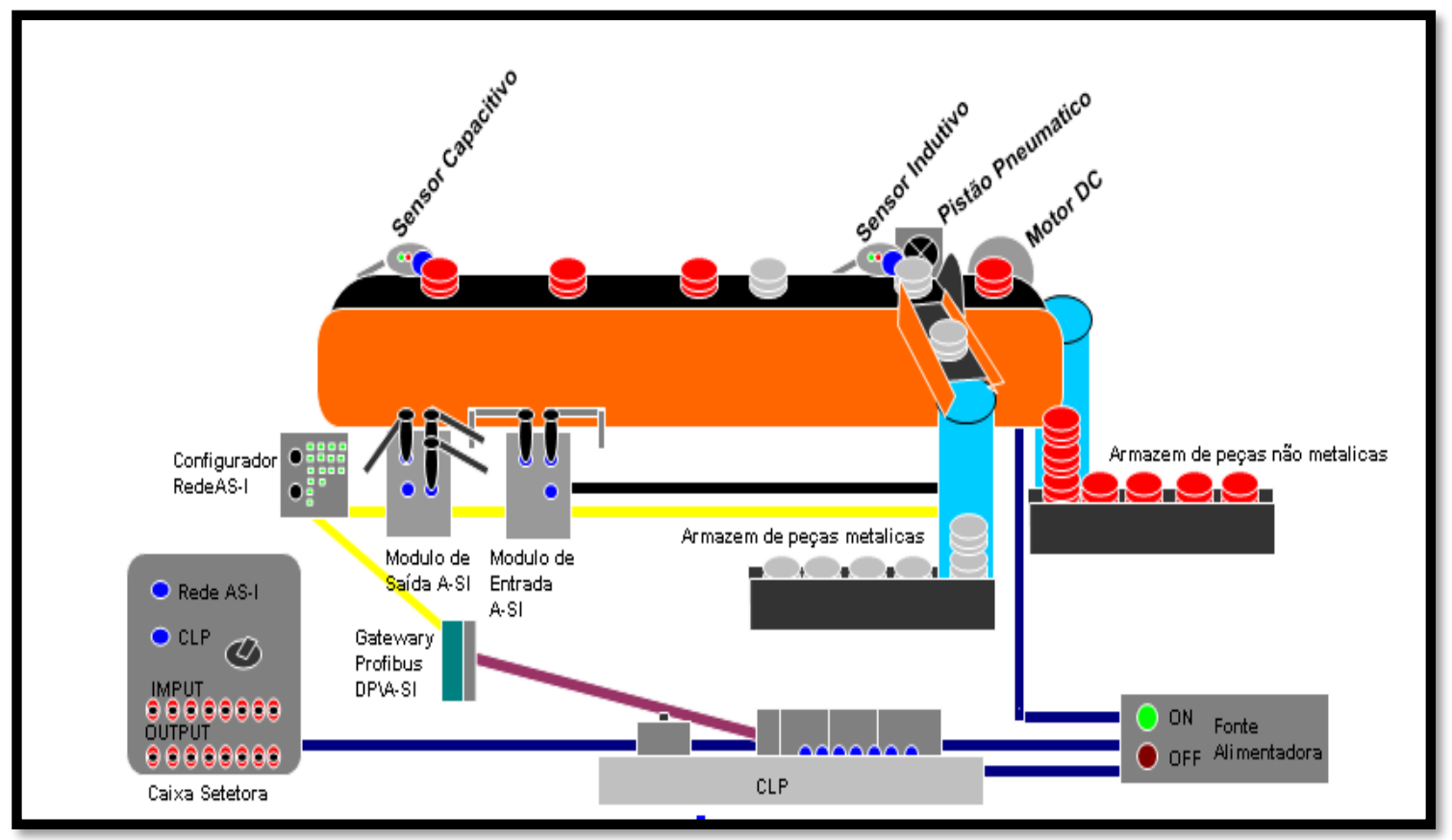

Fonte:Autoria Própria (2021).

O protótipo é constituído de módulos, acoplados a estrutura de uma esteira, configurados como entrada e saída de uma rede AS-I ${ }^{1}$, que fazem a leitura dos sinais dos sensores e direciona as atividades que os atuadores devem seguir de acordo com a programação do CLP.

Presentes também na bancada, há um motor de corrente contínua de $12 \mathrm{~V}$ (que movimenta a esteira), sensores indutivo e capacitivo, usados para identificar peças plásticas e metálicas, e um pistão pneumático, que é utilizado para separar as peças. Os sensores estão posicionados nas extremidades da esteira da bancada, com o propósito de detectar a passagem das peças, o primeiro sensor verifica se há a existência da peça, habilitando o acionamento do motor da esteira, movendo-a até o próximo sensor que identifica o material de composição da peça (metal ou plástico). Caso seja uma peça metálica, o pistão pneumático à expulsa para um reservatório, caso contrário, ela continua até o final da esteira onde será armazenada em outro reservatório. Se não houver a detecção da peça, a esteira se mantem em repouso. A Figura 2 apresenta o protótipo já finalizado.

A construção do experimento tem por base o projeto de aplicação de sistemas supervisório e de redes industriais, elaborado em MOREIRA et al. (2014), o qual foi referência para início das atividades e escolha para aplicação dos perfis de redes AS-I e

${ }^{1}$ Atuador Sensor Interface. 
Profibus DP, que apresentam relevância no contexto de aplicação da atividade laboral do engenheiro de automação.

Figura 2 - Bancada de Redes Industriais.
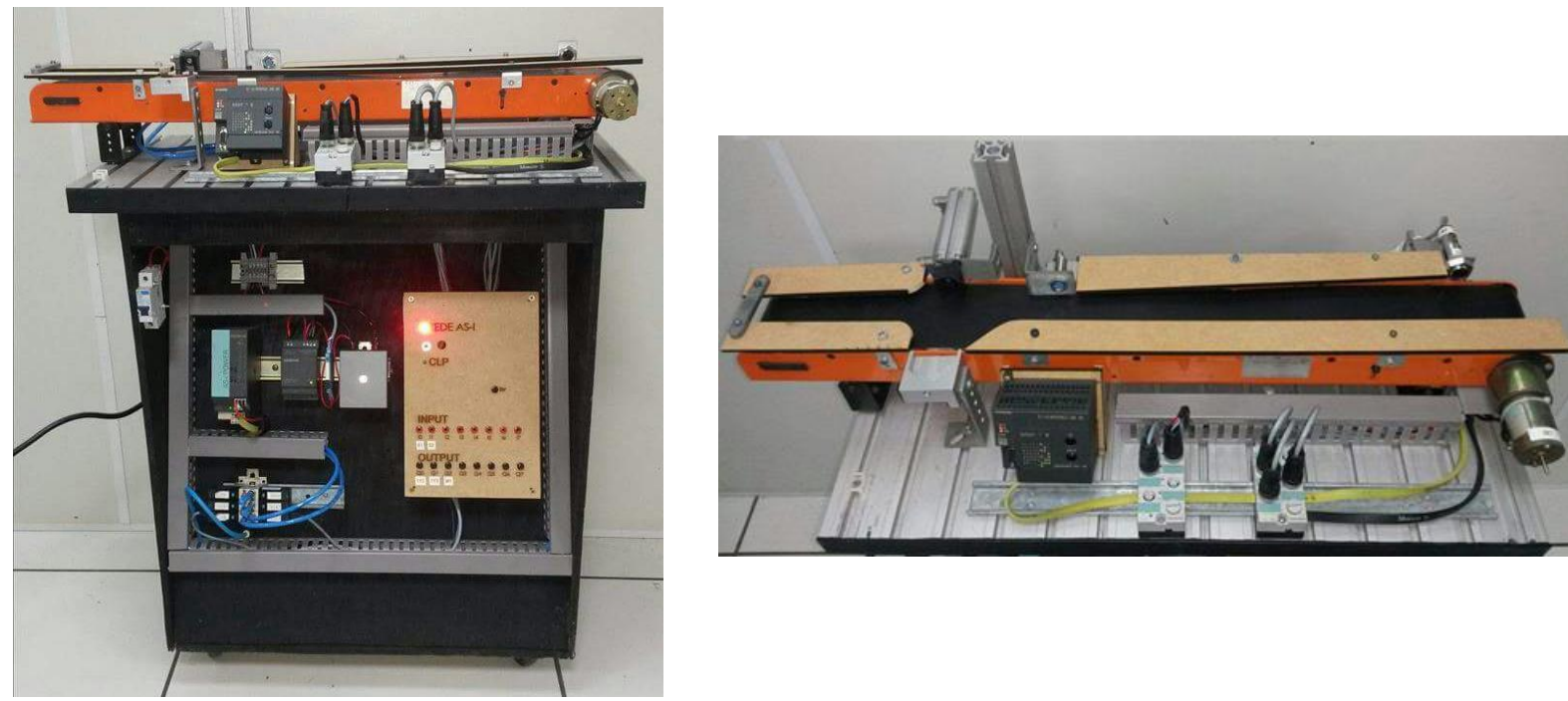

Fonte: Autoria Própria (2021).

Para que se de o início de processo de funcionamento da bancada e necessário o conjunto de componentes atuando de maneira cíclica e continua. Neste caso os principais componentes utilizados na integração da bancada em rede foram: um gateway Profibus DP/AS-Interface link 20E, uma fonte AS-I Power Supply e dois blocos I/O AS-I, para integração e montagem da rede AS-I, além de um CLP como mestre Profibus DP. Conforme apresentado na Figura 3.

Figura 3- Arquitetura das redes Profibus e AS-I empregados aos dispositivos da bancada.

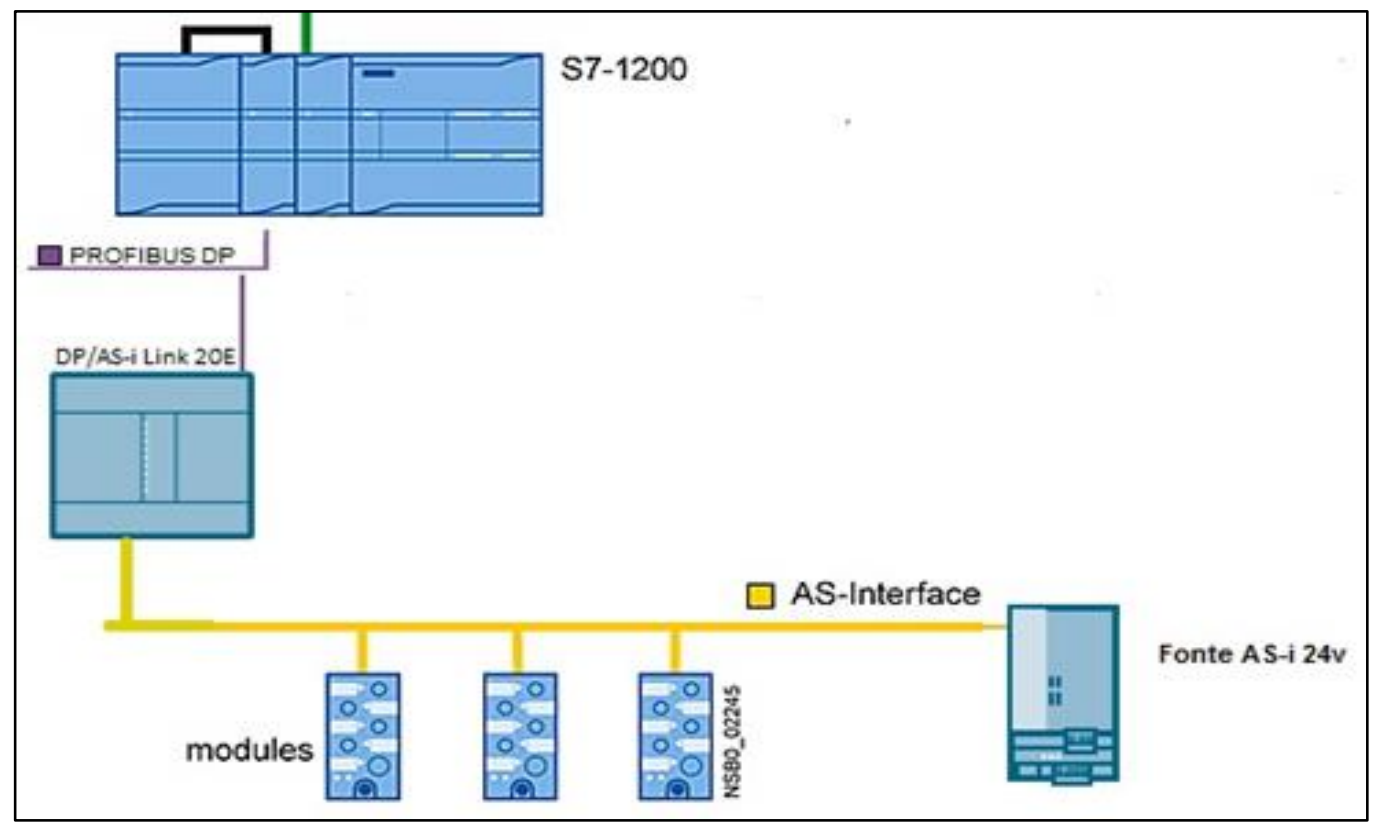

Fonte: Autoria Própria (2021). 
A programação e configuração da rede é realizada com base na leitura dos sinais do sensor e troca de informações via rede Profibus e AS-I, os quais são protocolos de redes industriais voltados a automação de processos distribuídos (LUGLI, 2010). Neste caso, foi utilizado o perfil de comunicação Profibus DP, que é empregado para substituir sistemas centralizados com CLPs em automação de processos de manufatura. No experimento, o Profibus DP funciona como um intermediário para que os sinais da rede AS-I cheguem ao CLP, uma vez que se utiliza um gateway, que funciona com um tradutor entre os protocolos de comunicação Profibus DP para rede AS-I, e vice-versa. O gateway e a rede Profibus DP, por sua vez, servem para a interligação da rede AS-I com nível hierárquico superior com o propósito de aumento a velocidade de tráfego de informações no sistema (LUGLI, 2010). Na sequência apresenta-se um exemplo de configuração de rede realizado na bancada objeto do estudo desse trabalho.

\section{$3 \quad$ RESULTADOS E DISCUSSÕES}

Como resultado principal, apresenta-se um exemplo de configuração do protótipo didático, que pode ser amplamente utilizado em aulas da área de engenharia de controle e automação. Assim, para configuração de rede é necessário a montagem física dos cabos e o arranjo de componentes de acordo com a aplicação que o sistema desempenha no projeto, como apresentado na arquitetura da rede AS-I aplicada na bancada na Figura 3.

Após a escolha da arquitetura, insere-se o gateway conectando através de rede Profibus DP num CLP Siemens S7-1200. Na sequência é configurado o software na plataforma de programação do CLP, onde configura-se o gateway no software de programação. Toda a configuração da rede Profibus DP é realizada no CLP, uma vez que este equipamento é o mestre dessa rede. Por outro lado, a configuração da rede AS-I é realizada manualmente, via um configurador AS-I (Figura 4) conectado individualmente a cada módulo de entrada/saída. Portanto, de uma forma bem mais ampla, o gateway é configurado como mestre da rede AS-I, ao mesmo tempo em que este equipamento é escravo numa rede Profibus DP.

Assim, configuraram-se inicialmente os módulos de entrada e saída utilizando um configurador AS-I. A Figura 4 apresenta o configurador AS-I e um módulo de entrada com seu respectivo endereço de rede.

Figura 4 - Módulo de entrada endereçado a partir de um Configurador AS-I.

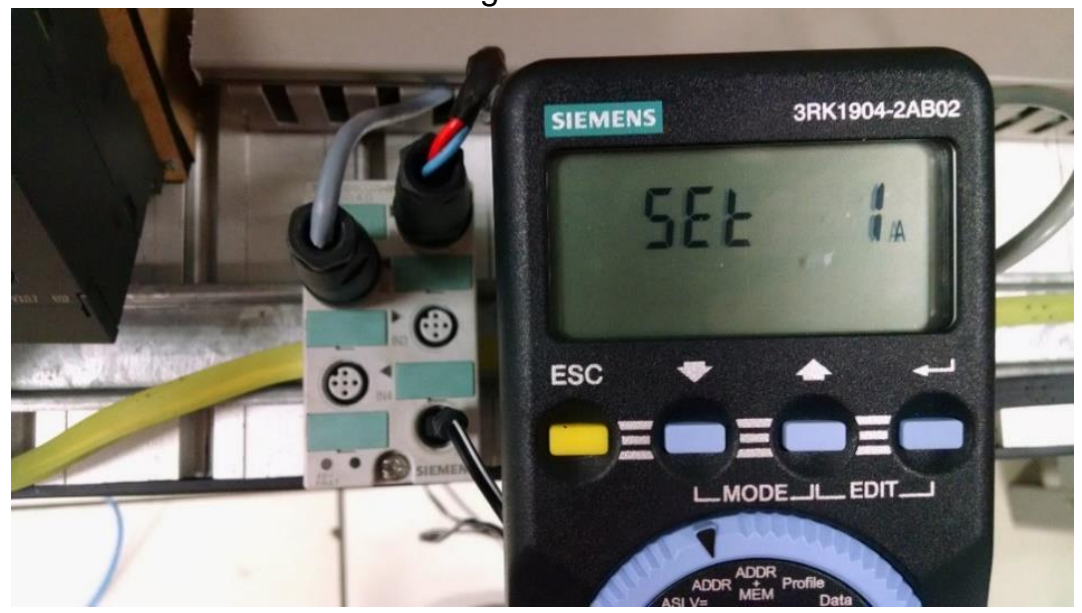

Fonte: Autoria Própria (2021). 
Após configurado dos módulos, volta-se a configuração do gateway que faz a ponte entre as redes Profibus-DP e AS-I. Usou-se o endereço 4 para o gateway na rede Profibus DP, como apresenta a Figura 5.

Figura 5 - Gateway Profibus DB/AS-I.

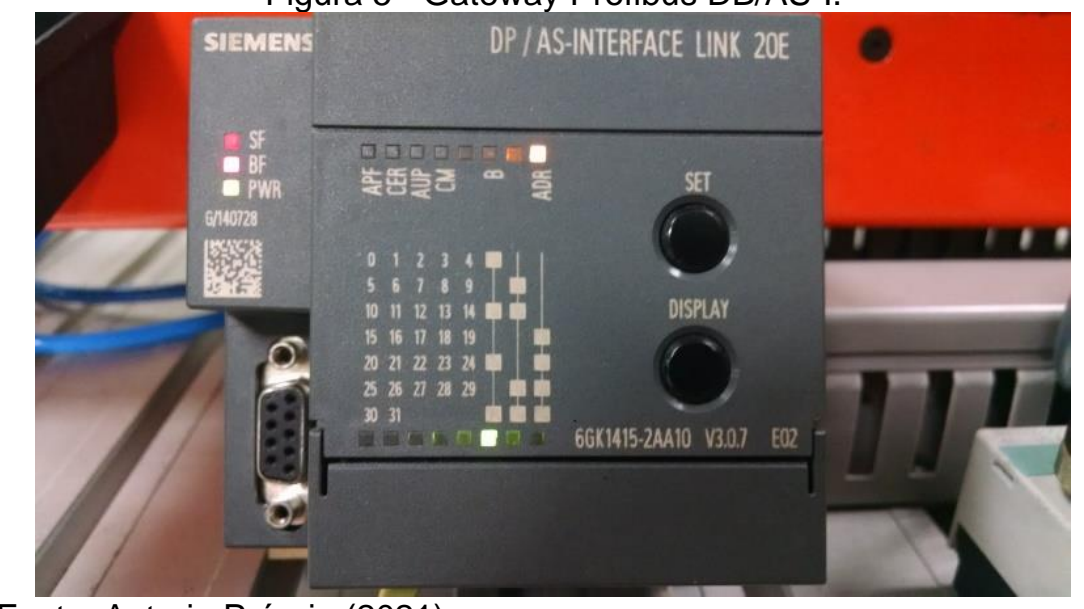

Fonte: Autoria Própria (2021).

Além das configurações de hardware, é necessário também realizar as configurações no software, relacionadas a inserção dos equipamentos utilizados, seus respectivos endereços de comunicação, assim como a lógica de funcionamento para os componentes instalados na rede. As Figuras 6 e 7 mostram as conexões e os endereços dos equipamentos no software de configuração do CLP.

Figura 6 - Conexões da rede Profibus DP no software de configuração do CLP.

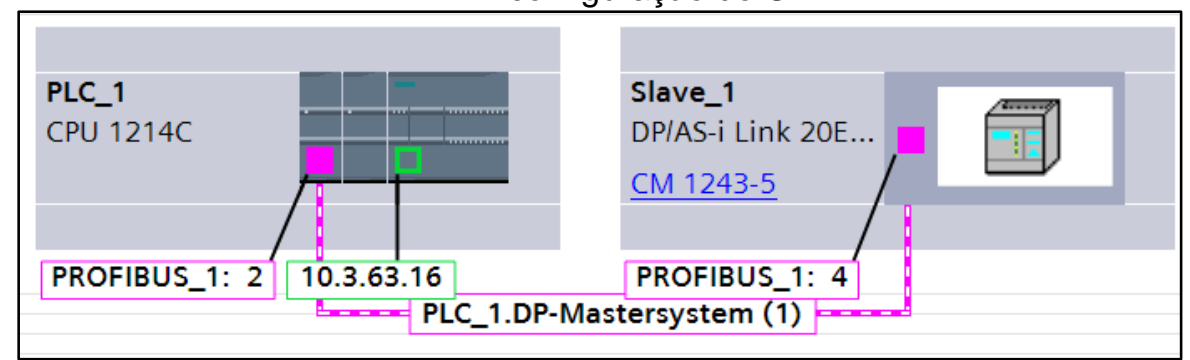

Fonte: Autoria Própria (2021).

Figura 7 - Configuração do gateway Gateway Profibus DB/AS-I no software de configuração do CLP.

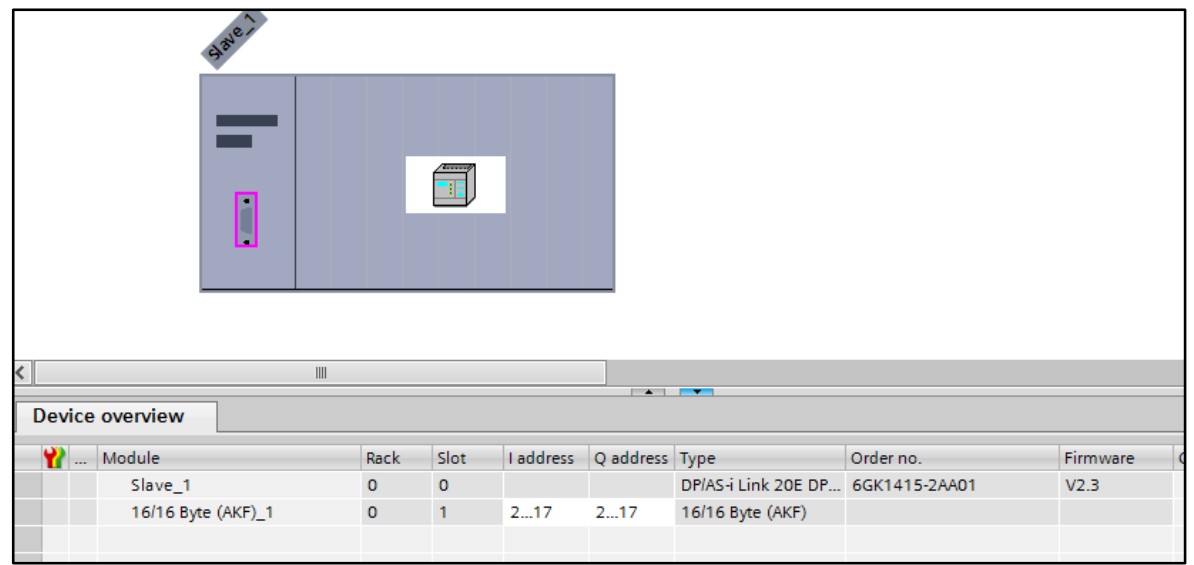

Fonte: Autoria Própria (2021). 
A Figura 7 apresenta os respectivos endereços de entrada e saída atribuídos a tabela de endereços AS-I do gateway. Essa relação de endereços é observada a partir da Figura 8 (SIEMENS, 2008).

Figura 8 - Tabela de Endereços de Entrada e Saída Profibus atribuídos aos respectivos endereços AS-I.

\begin{tabular}{|c|c|c|}
\hline Byte Number *) & Bit 7-4 & Bit 3-0 \\
\hline$m+0$ & Status Nibble ${ }^{* *}$ ) & $\begin{array}{l}\text { Slave } 1 \text { or } 1 \mathrm{~A} \\
\text { Bit } 3 \text { | Bit } 2 \text { |Bit } 1 \text { | Bit } 0\end{array}$ \\
\hline$m+1$ & Slave 2 or $2 \mathrm{~A}$ & Slave 3 or $3 \mathrm{~A}$ \\
\hline$m+2$ & Slave 4 or $4 \mathrm{~A}$ & Slave 5 or $5 \mathrm{~A}$ \\
\hline$m+3$ & Slave 6 or $6 \mathrm{~A}$ & Slave 7 or $7 \mathrm{~A}$ \\
\hline$m+4$ & Slave 8 or $8 \mathrm{~A}$ & Slave 9 or $9 \mathrm{~A}$ \\
\hline$m+5$ & Slave 10 or $10 \mathrm{~A}$ & Slave 11 or $11 \mathrm{~A}$ \\
\hline$m+6$ & Slave 12 or $12 \mathrm{~A}$ & Slave 13 or $13 \mathrm{~A}$ \\
\hline$m+7$ & Slave 14 or $14 \mathrm{~A}$ & Slave 15 or $15 \mathrm{~A}$ \\
\hline$m+8$ & Slave 16 or $16 \mathrm{~A}$ & Slave 17 or $17 \mathrm{~A}$ \\
\hline$m+9$ & Slave 18 or $18 \mathrm{~A}$ & Slave 19 or $19 \mathrm{~A}$ \\
\hline$m+10$ & Slave 20 or $20 \mathrm{~A}$ & Slave 21 or $21 \mathrm{~A}$ \\
\hline$m+11$ & Slave 22 or $22 \mathrm{~A}$ & Slave 23 or $23 \mathrm{~A}$ \\
\hline$m+12$ & Slave 24 or $24 \mathrm{~A}$ & Slave 25 or $25 \mathrm{~A}$ \\
\hline
\end{tabular}

Fonte: Autoria Própria (2021).

Assim, de acordo com a tabela na Figura 8, as entradas e saídas a serem acionados para funcionamento correto da rede, de acordo com os sensores e atuadores conectados fisicamente, são: 12.0 (Sensor indutivo), 12.1 (Sensor capacitivo), Q3.5 (Avanço do atuador pneumático), Q3.6 (Funcionamento do motor) e Q3.7 (Retorno do atuador pneumático). Os endereços apresentados fazem parte da tabela de entradas e saídas do CLP, o que mostra que para programação do CLP e da lógica do algoritmo, a rede é vista como módulos de entrada e saída remotos do CLP.

Figura 9 - Fluxograma de execução atividades.

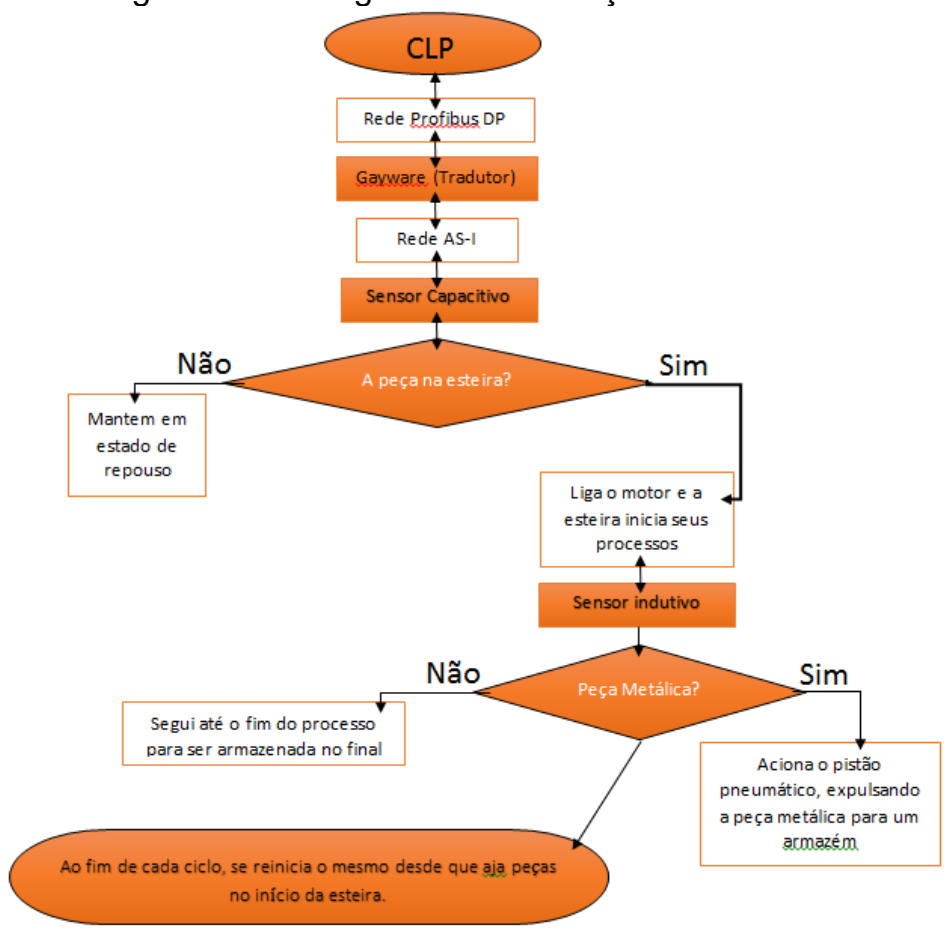

Fonte: Autoria Própria (2021). 
Assim, o fluxograma da Figura 9 mostra como a programação do CLP define a execução de cada atividade. Dada à configuração da rede, os dispositivos periféricos (componentes da rede AS-I) são reconhecidos na programação do CLP como endereços de entradas e saídas. Assim, a Figura 9, apresenta de forma simplificada o algoritmo de funcionamento da programação do processo, utilizando dados coletados (endereços de entrada e saída) pelo configurador de rede AS-I.

Observa-se que para a inicialização, configuração e programação do processo é fundamental que o estudante busque as informações de diferentes fontes e conceitos, necessárias para integração do sistema, como programação de CLPs, configuração de redes de comunicação, instrumentação, eletrônica digital e circuitos elétricos, caracterizando a multidisciplinaridade quanto a aplicação do protótipo didático, ao mesmo tempo em que a construção do experimento apresenta caráter interdisciplinar, mostrandose uma boa ferramenta para o ensino de engenharia de automação. Nesse sentido, no Apêndice A apresenta-se um exemplo de aplicação do sistema, integrado a um robô, simulando uma célula de automação, comum nas futuras atividades do egresso de cursos da área.

\section{CONCLUSÃO}

O protótipo didático apresentado tem a proposta de inicializar o estudante ao ambiente de trabalho do engenheiro de automação, proporcionando um aprendizado tanto técnico como teórico, a partir de uma metodologia de ensino ativa empregada, tanto nas fases de construção quanto na operação do sistema.

Assim, a utilização da dessa ferramenta didática se mostra uma opção prática para ensino de automação a estudantes da área de engenharia, tornando o ensino mais atraente e instigando os discentes na busca de conhecimento, uma vez que para a construção, configuração e programação do sistema apresentado necessita-se da aplicação de conceitos de diversas disciplinas, e permite ao estudante a obtenção de experiência através da análise de resultados em sistemas similares aos encontrados no mercado de trabalho, considerando questões como custos, manutenções e viabilidade de implementação.

Como desenvolvimentos de atividades futuras, busca-se a integração da bancada com outros processos de automação (via protocolos industriais) de modo a desenvolver um processo de manufatura integrado a um sistema supervisório para monitoramento remoto. Dessa forma, aproximando o estudante a um processo de automação industrial 4.0.
Apêndices
Apêndice A.
Vídeo de demonstração do protótipo didático: https://youtu.be/i5BDbl3vO3E .

\section{REFERÊNCIAS}

\section{BENDER, W. N. Aprendizagem baseada em projetos, educação diferenciada para o} século XXI. 1. ed. São Paulo: PENSO Editora, 2014.

BRASIL. Ministério da Educação. Resolução no 2, de 24 de abril de 2019. Institui as diretrizes curriculares nacionais do curso de graduação em engenharia. Diário Oficial da União. Brasília, DF, 24 abr. 2019. Disponível em: https://www.in.gov.br/web/dou/- 
/resolu\%C3\%87\%C3\%83o-n\%C2\%BA-2-de-24-de-abril-de-2019-85344528. Acesso em: 20 abr. 2021.

LUGLI, Alexandre Baratella; SANTOS, Max Mauro Dias. Redes industriais para automação industrial: AS-I, PROFIBUS e PROFINET. São Paulo (SP): Érica. 2011.

NASCIMENTO, Vivyane Alencar Marques Araújo do. O USO DO CONTROLADOR LÓGICO PROGRAMÁVEL (CLP). In: MARTINS, Ernane Rosa (org.). Tecnologias, Métodos e Teorias na Engenharia da Computação. Ponta Grossa: Atena Editora, 2020. Cap. 7, p. 59. Disponível em:

https://www.finersistemas.com/atenaeditora/index.php/admin/api/artigoPDF/41930. Acesso em: 20 abr. 2021.

MILHOMEM, R. L.; BAYMA, R. S.; CASTRO, A. M.; SILVA, C. D. Desenvolvimento de um protótipo didático de levitação magnética. In: XXXVII - Congresso Brasileiro de Ensino de Engenharia, 2009, Recife. Anais. Recife. Disponível em:

http://www.abenge.org.br/cobenge.php. Acesso em 15 de abr. 2021.

MOREIRA, B. A.; SILVA, J. J.; PERKUSICH, A; NETO, J. S. R.. Uso de sistemas supervisórios para o estudo das redes industriais asi e profibus. In: XLII - Congresso Brasileiro de Ensino de Engenharia, 2014, Juiz de Fora. Anais. Juiz de Fora. Disponível em: http://www.abenge.org.br/cobenge.php . Acesso em 20 de abr. 2021.

SIEMENS. AS-Interface/AS-Isafe. System Manual, 2008. Disponivel em: https://promspecrele.ru/documents/AS-Interface System\%20Manual 2008-11.pdf . Acesso em: 09 abr. 2021.

\title{
DISCUSSIONS ON THE DEVELOPMENT AND USE OF A TEACHING PROTOTYPE FOR TEACHING INDUSTRIAL NETWORKS
}

\begin{abstract}
The need for qualified professionals to work with technologies for automation of industrial processes has been growing and becoming increasingly imperative due to the integration of information and the increasing computerization of these processes. In this case, it is necessary to have quality technical training that develops the practical and theoretical integration of knowledge in an attractive way. In this context, a prototype of an Industrial Network Bench was built, aiming at better presenting knowledge in the teaching of automation, integrating the disciplines of Communication Networks and Programming of Programmable Logic Controllers. Finally, it discusses the construction of prototypes such as the Bench of Industrial Networks, as a didactic and attractive form of technical education, presenting future professionals to their possible work routines.
\end{abstract}

Key-words: Industrial Networks, Intergeneration of Systems, Didactic Prototype. 